\begin{tabular}{|r|l|}
\hline \multicolumn{2}{|c|}{ Statistica Sinica Preprint No: SS-2020-0429 } \\
\hline Title & $\begin{array}{l}\text { Model Checking for Parametric Ordinary Differential } \\
\text { Equations Systems }\end{array}$ \\
\hline Manuscript ID & SS-2020-0429 \\
\hline URL & http://www.stat.sinica.edu.tw/statistica/ \\
\hline DOI & $10.5705 /$ ss.202020.0429 \\
\hline Complete List of Authors & $\begin{array}{l}\text { Ran Liu, } \\
\text { Yun Fang and } \\
\end{array}$ \\
Lixing Zhu \\
\hline Corresponding Author & Lixing Zhu \\
\hline E-mail & lzhu@hkbu.edu.hk \\
\hline Notice: Accepted version subject to English editing. \\
\hline
\end{tabular}




\title{
Model Checking for Parametric Ordinary Differential Equations Systems
}

\author{
Ran Liu, Yun Fang and Lixing Zhu \\ Hong Kong Baptist University, Shanghai Normal University \\ and Beijing Normal University at Zhuhai
}

Abstract: Model checking for parametric ordinary differential equations is a necessary step to check whether the assumed models are plausible. In this paper, we first introduce a trajectory matching-based test for the whole model, which can also easily be applied to check partially observed systems. Then we provide two tests to identify which component function would be wrongly modelled. The first is integral matching-based, and the second is gradient matching-based with a bias correction achieved by data splitting. We investigate their asymptotic properties under the null, global and local alternative hypotheses. As there are no results of relevant parameter estimations with the alternative models in the literature, we also investigate the asymptotic properties of nonlinear least squares estimation and two-step estimation under both the null and alternatives. To examine the performances of the tests, we conduct several numerical simulations and the analysis for a real data example about immune cell kinetics and trafficking for influenza infection. 
Key words and phrases: Local smoothing test; Model checking; Ordinary differential equations.

\section{Introduction}

For modelling how the systems evolve with time, ordinary differential equations (ODEs) have been widely applied in many scientific fields such as physics, ecology (Goel et al. (1971)) and neuroscience (FitzHugh (1961); Nagumo et al. (1962)). A system of ODEs can be written as

$$
X^{\prime}(t)=\left[\begin{array}{c}
\frac{d X_{1}(t)}{d t} \\
\vdots \\
\frac{d X_{p}(t)}{d t}
\end{array}\right]=\left[\begin{array}{c}
f_{1}(t, X(t) ; \theta) \\
\vdots \\
f_{p}(t, X(t) ; \theta)
\end{array}\right]=f(t, X(t) ; \theta), \quad t \in\left[t_{0}, T\right]
$$

with an initial condition $X\left(t_{0}\right)=x_{0}$. Here $X(t)=\left(X_{1}(t), \ldots, X_{p}(t)\right)^{\top}$ is a $p$-dimensional state vector and $f(t, X(t) ; \theta)$ is supposed to belong to a given parametric family of functions $\mathcal{F}=\left\{f(\cdot, \theta): \theta \in \Theta \subset R^{q}\right\}$. Frequently, this system is measured on discrete time points with noises, say

$$
Y_{i}=X\left(t_{i}\right)+\epsilon_{i}, \quad i=1, \ldots, n
$$

where the measurement error $\epsilon_{i}$ satisfying $E\left(\epsilon_{i} \mid t_{i}\right)=0$ has nonsingular variance-covariance matrix $\Sigma_{\epsilon_{i}}$, and is independent with $\epsilon_{j}$ for every $j \neq i$. The observation process may be more complicated in real-life applications. 
For example, the observable variable might be a combination or a functional of state variables. In this paper, we focus on the simple observation process case represented by 1.2 .

In this paper, we want to check:

$$
H_{0}: X^{\prime}(t)=f\left(t, X(t) ; \theta_{0}\right) \in \mathcal{F} \quad \text { versus } \quad H_{1}: X^{\prime}(t) \notin \mathcal{F},
$$

where $\theta_{0}$ is an unknown parameter vector. If we reject $H_{0}$, we may further wish to identify the component(s) that would be wrongly modelled. In this situation, for the $k$-th component function, the hypotheses are as follows,

$$
H_{0 k}: X_{k}^{\prime}(t)=f_{k}\left(t, X(t) ; \theta_{0 k}\right) \in \mathcal{F}_{k} \quad \text { versus } \quad H_{1 k}: X_{k}^{\prime}(t) \notin \mathcal{F}_{k},
$$

with $\mathcal{F}_{k}=\left\{f_{k}\left(\cdot, \theta_{k}\right): \theta_{k} \in \Theta_{k} \subset R^{q_{k}}\right\}$. In the current paper, we construct omnibus tests for checking the whole parametric ODE system (1.3) and individual components 1.4 .

To this end, we first review some relevant methodologies of model checking for classical regressions in the literature which motivate the new test constructions we need. In the univariate response cases, there exist two broad classes of tests. The so-called local smoothing tests are constructed by using nonparametric estimations include Härdle and Mammen (1993), Zheng (1996), Dette (1999) and Lavergne and Patilea (2012) as examples. Empirically, tests in this class are sensitive to alternative models that are 
oscillating/highly frequent in general. Tests in another class are based on residual-marked empirical processes and take averages over an index set. As averaging itself is a global smoothing step, they are called global smoothing tests. See relevant references such as Stute (1997), Stute et al. (1998), Zhu (2003) and Khmaladze and Koul (2004). Such tests have better asymptotic properties, but are less sensitive to oscillating alternative models. González-Manteiga and Crujeiras (2013) gave a comprehensive review. For multi-response regressions, Chen and van Keilegom (2009) constructed an empirical likelihood ratio test based on local smoothing.

The testing problems investigated in the current paper are however rather different from those for classical parametric regression models as the model structure is not directly assumed on the unknown function $X(\cdot)$, but on its derivative $X^{\prime}(\cdot)$, and even only part of the whole ODE system is observed. Further, any component of $X^{\prime}(\cdot)$ is related to the whole original function $X(\cdot)$ rather than any single corresponding component of $X(\cdot)$. This structure then causes the testing problems complicated. We will discuss these issues in the following sections. In the literature, to the best of our knowledge, there are only two relevant references available concerning the ODE model checking problem. Hooker (2009) proposed a goodness-offit test based on estimated forcing functions for the whole ODE system. 
They transferred the ODE system to a multivariate linear model under the null hypothesis against this model adding an empirical forcing function represented as a basis expansion under the alternative hypothesis, then used a likelihood-ratio test to check the whole ODE system. Under the normality, independent components and homoscedasticity assumptions on error terms when the transferred semiparametric model can be regarded as a mixedeffects model, its null distribution is tractable. The asymptotic properties under the global and local alternatives remained unknown. For the nonlinear null ODE models, they discussed an extension in the sense that the test is based on a linear approximation that distorts the null distribution of the test. This test cannot identify which individual components would be wrongly modelled. Without the regularity assumptions mentioned above, the asymptotic properties were not investigated. Hooker and Ellner (2015) gave some further discussion.

In this paper, we will construct three tests. The first test is for checking the whole ODE system (1.3). It is based on the trajectory representation of ODEs that solves (1.1) and represents the ODE system as a multi-response function of time:

$$
X(t)=F(t ; \theta)
$$

where $F(t ; \theta)$ denotes the trajectory solution of 1.1$)$. This test is con- 
structed by matching approximations of two sides of 1.5 , thus is called a trajectory matching-based test $\left(T M_{n}\right)$. It is also feasible for partially observed ODE systems (Dattner $(2015))$ of which some components are not measured. However, we will show the trajectory matching-based test fails to check each individual component (1.4) due to mixed components and parameters effects. Thus, we consider two other tests for testing the components (1.4). We construct an integral matching-based test $\left(I M_{n}\right)$ that is based on the integration representation of ODEs: on the definite integral over the interval $\left(t_{0}, t\right)$ for both sides of 1.1$)$ :

$$
X(t)-X\left(t_{0}\right)=\int_{t_{0}}^{t} f(s, X(s) ; \theta) d s .
$$

Although this integral matching-based test can be of consistency in theory, its empirical performance is not encouraging due to the cumulative error in the integral and estimation error in nonparametric estimation involved. Therefore, we also construct a gradient matching-based test $\left(G M_{n}\right)$ that is directly based on the gradient representation of ODEs (1.1), but with a data splitting technique to eliminate the bias. We then call it a bias-corrected gradient matching-based test. The new tests are all local smoothing-type because some useful ODE models are highly oscillating, such tests may better detect possible model departures from the null model.

As for parameters estimation, we will use the nonlinear least squares 
method that is based on the trajectory representation 1.5 for $T M_{n}$. See Xue et al. (2010) and Ramsay and Hooker (2017) for details. However, for checking each component function, this estimation fails to work because it involves all components of the system. Thus, the two-step estimation methods are considered. They first replace the unknown functions in the integral representation $(1.6)$ or the gradient representation 1.1 with their nonparametric estimators, and then construct pseudo-least squares estimators. The relevant references include Brunel (2008), Liang and $\mathrm{Wu}$ (2008) and Dattner and Klaassen (2015). We will use a gradient matching two-step estimation method for $I M_{n}$ and $G M_{n}$. Note the estimation methods based on three model representations actually pair with the three proposed tests.

In the rest of this paper, we will give the detailed constructions for the three tests and study their asymptotic properties under the null, global and local alternatives. We will also investigate the properties of the corresponding estimators under both the null and alternative hypotheses, which is also new to the best of our knowledge. The technical proofs are postponed to Supplementary Material. In this paper, the measurement time $t_{i}$ is considered random with the sampling probability density function $p(t)$, which is a convenient mathematical device used in some literatures about ODE models such as Liang and Wu (2008) and Ding and Wu (2014). 


\section{Trajectory matching-based test}

\subsection{Test statistic construction}

Recall the hypotheses in $(1.3)$ for the whole ODE system. According to the trajectory representation 1.5 , the checking problem can be converted to testing whether the vector function $X(t)=F\left(t ; \theta_{0}\right)$ for some $\theta_{0} \in \Theta \subset R^{q}$.

Consider the $p=1$ case to motivate our construction. Recall $p(t)$ denotes the sampling density of the measurement time $t_{i}$. Denote $\|\cdot\|$ as the Frobenius norm. Let $\varepsilon_{i}=Y_{i}-F\left(t_{i} ; \theta_{N L S}^{*}\right)$ with $\theta_{N L S}^{*}=\arg \min _{\theta} E\left\{\left\|Y_{i}-F\left(t_{i} ; \theta\right)\right\|^{2}\right\}$. Under $H_{0}, \varepsilon_{i}=\epsilon_{i}$ and $E\left(\varepsilon_{i} \mid t_{i}\right)=0$ leads to $E\left\{\varepsilon_{i} E\left(\varepsilon_{i} \mid t_{i}\right) p\left(t_{i}\right)\right\}=0$, while under $H_{1}, E\left(\varepsilon_{i} \mid t_{i}\right)=X\left(t_{i}\right)-F\left(t_{i} ; \theta_{N L S}^{*}\right) \neq 0$, and $E\left\{\varepsilon_{i} E\left(\varepsilon_{i} \mid t_{i}\right) p\left(t_{i}\right)\right\}=$ $E\left[\left\{E\left(\varepsilon_{i} \mid t_{i}\right)\right\}^{2} p\left(t_{i}\right)\right]>0$. With the nonlinear least squares estimator $\hat{\theta}_{N L S}=\arg \min _{\theta} \sum_{i=1}^{n}\left\|Y_{i}-F\left(t_{i} ; \theta\right)\right\|^{2}, e_{i}=Y_{i}-F\left(t_{i} ; \hat{\theta}_{N L S}\right)$ is the residual. Thus, we use the sample analogue of $E\left\{\varepsilon_{i} E\left(\varepsilon_{i} \mid t_{i}\right) p\left(t_{i}\right)\right\}$ to build a statistic

$$
V_{n}^{Z h}=\frac{1}{n(n-1)} \sum_{i=1}^{n} \sum_{\substack{j=1 \\ j \neq i}}^{n} \frac{1}{h} K\left(\frac{t_{i}-t_{j}}{h}\right) e_{i} e_{j},
$$

where $K$ is a kernel function, $h$ is a bandwidth parameter. This is in spirit similar to the test suggested by Zheng (1996). A standardized test statistic $T_{n}^{Z h}$ can be easily obtained by using $V_{n}^{Z h}$ and its variance. In the multi-response case, we can obtain a vector version of $V_{n}^{Z h}$ as $V_{n}^{F}=$ $\left(V_{n 1}^{Z h}, \ldots, V_{n p}^{Z h}\right)^{\top}$. To summarize the information contained in $V_{n}^{F}$, we ag- 
gregate $V_{n}^{F}$ to make a test statistic and write it as $T M_{n}$ in short:

$$
T M_{n}=n^{2} h V_{n}^{F^{\top}}\left(\widehat{\Sigma}^{F}\right)^{-1} V_{n}^{F}
$$

Here $\widehat{\Sigma}^{F}$ is a symmetric matrix used to normalize the test statistic:

$$
\widehat{\Sigma}^{F}=\frac{2}{n(n-1)} \sum_{i=1}^{n} \sum_{\substack{j=1 \\ j \neq i}}^{n} \frac{1}{h} K^{2}\left(\frac{t_{i}-t_{j}}{h}\right)\left(e_{i} \odot e_{j}\right)\left(e_{i} \odot e_{j}\right)^{\top}
$$

where $\odot$ denotes the element-wise product of two vectors and $e_{i}$ is the residual vector at the time point $t_{i}$. By using quadratic form and taking $\left(\widehat{\Sigma}^{F}\right)^{-1}$ for normalization, we eliminate the correlation among components and place equal weight on them.

In practice, it is often that the data are only available for a subset of components of the ODE system $(\overline{\text { Dattner }}(2015))$. For instance, we can not observe the susceptible population in the classical SIR model for infectious diseases. The proposed test $T M_{n}$ can be easily extended to a modified test $T M_{n}^{o}$ to handle this case by replacing the statistics of all components by their corresponding statistics of those observed components. See Supplementary Material for details.

\section{$2.2 \quad$ Asymptotic properties}

To derive the asymptotic properties of $T M_{n}$, we suppose sets $\mathrm{A}$ and $\mathrm{B}$ of assumptions in Supplementary Material hold. The assumptions in set A 
give the basic setting of $(t, Y)$ and the conditions on the kernel function and $X^{\prime}(t)$. The assumptions in set $\mathrm{B}$ place restrictions on the primitive function $F(t ; \theta)$, which are about the model identifiability for the nonlinear least squares estimation (see, e.g. White (1981)).

To investigate the power of a test, we consider two kinds of local alternatives for ODE models later. The first one adds local misspecification to the trajectory of ODEs:

$$
H_{1 n}^{F}: X(t)=F\left(t ; \theta_{0}\right)+\delta_{n} L(t)
$$

where $L(t)=\left(L_{1}(t), \ldots, L_{p}(t)\right)^{\top}$ is a bounded multiple response function such that $X(t) \neq F(t ; \theta)$ for every $\theta \in \Theta$, and $\delta_{n} \rightarrow 0$ as $n \rightarrow \infty$. $H_{1 n}^{F}$ in (2.7) is similar as in the classical regression settings. However, things are more complicated for the ODE models: another type of local disturbance is directly on the derivative function $X^{\prime}(\cdot)$. That is, we consider the following sequence of local alternatives:

$$
H_{1 n}^{f}: X^{\prime}(t)=f\left(t, X(t) ; \theta_{0}\right)+\delta_{n} l(t)
$$

where $l(t)=\left(l_{1}(t), \ldots, l_{p}(t)\right)^{\top}$ is a bounded multiple response function and $X^{\prime}(t) \notin \mathcal{F}$. Hooker $(2009)$ considered global alternatives in a similar manner by using empirical forcing functions. We now state the relationship between $H_{1 n}^{f}$ and $H_{1 n}^{F}$. 
Proposition 1. Given the set A of assumptions in Supplementary Material, then under $H_{1 n}^{F}$ in (2.7), the derivative has the form

$$
X^{\prime}(t)=f\left(t, X(t) ; \theta_{0}\right)+\delta_{n} v_{1}(t)+o\left(\delta_{n}\right) .
$$

where $v_{1}(t)=L^{\prime}(t)-\frac{\partial f\left(t, X(t) ; \theta_{0}\right)}{\partial X^{\top}} L(t)$. Under $H_{1 n}^{f}$ in 2.8), the original function can be expressed as

$$
X(t)=F\left(t ; \theta_{0}\right)+\delta_{n} v_{2}(t)+o\left(\delta_{n}\right),
$$

where $v_{2}(t)$ is the solution of $v_{2}^{\prime}(t)=\frac{\partial f\left(t, X(t) ; \theta_{0}\right)}{\partial X^{\top}} v_{2}(t)+l(t)$ with $v_{2}(0)=0$.

As any higher order little $o$ term does not influence the asymptotic properties of the test under the local alternatives, $H_{1 n}^{F}$ is asymptotically equivalent to $H_{1 n}^{f}$ in this sense. But in finite sample cases, they may still affect the performances of the tests. In the following, we define $l(t)=v_{1}(t)$ under $H_{1 n}^{F}$ and $L(t)=v_{2}(t)$ under $H_{1 n}^{f}$, then uniformly handle these two kinds of local alternatives.

Before exploring the limiting results of $T M_{n}$, we first study the asymptotic properties of the nonlinear least squares estimator. Recall $\theta_{N L S}^{*}=$ $\arg \min _{\theta} E\left\{\left\|Y_{i}-F\left(t_{i} ; \theta\right)\right\|^{2}\right\}$. We give the following proposition.

Proposition 2. Given sets $A$ and $B$ of assumptions in Supplementary Material, supposing the numerical error of numerical solution is negligible, then 
$\hat{\theta}_{N L S}-\theta_{N L S}^{*}=o_{P}(1)$. Also, we have the following.

1. Under the null hypothesis, $\theta_{N L S}^{*}=\theta_{0}$ and with

$$
H_{\dot{F}}=E\left\{\sum_{k=1}^{p} \frac{\partial F_{k}\left(t ; \theta_{0}\right)}{\partial \theta} \frac{\partial F_{k}\left(t ; \theta_{0}\right)}{\partial \theta^{\top}}\right\}
$$

then

$$
\sqrt{n}\left(\hat{\theta}_{N L S}-\theta_{0}\right)=H_{\dot{F}}^{-1} \frac{1}{\sqrt{n}} \sum_{i=1}^{n} \sum_{k=1}^{p}\left\{\epsilon_{i k} \frac{\partial F_{k}\left(t_{i} ; \theta_{0}\right)}{\partial \theta}\right\}+o_{P}(1) .
$$

2. Under the global alternative hypothesis $H_{1}$, writing $\theta_{N L S}^{*}=\theta_{1}^{*}$,

$$
\sqrt{n}\left(\hat{\theta}_{N L S}-\theta_{1}^{*}\right)=G_{\dot{F}}^{-1} \frac{1}{\sqrt{n}} \sum_{i=1}^{n} \sum_{k=1}^{p}\left[\left\{Y_{i k}-F_{k}\left(t_{i} ; \theta_{1}^{*}\right)\right\} \frac{\partial F_{k}\left(t_{i} ; \theta_{1}^{*}\right)}{\partial \theta}\right]+o_{P}(1)
$$

where

$G_{\dot{F}}=E\left\{\sum_{k=1}^{p} \frac{\partial F_{k}\left(t ; \theta_{1}^{*}\right)}{\partial \theta} \frac{\partial F_{k}\left(t ; \theta_{1}^{*}\right)}{\partial \theta^{\top}}\right\}-E\left[\sum_{k=1}^{p}\left\{X_{k}(t)-F_{k}\left(t ; \theta_{1}^{*}\right)\right\} \frac{\partial^{2} F_{k}\left(t ; \theta_{1}^{*}\right)}{\partial \theta \partial \theta^{\top}}\right]$.

3. Under the local alternative hypothesis $H_{1 n}^{F}$ in (2.7) or $H_{1 n}^{f}$ in (2.8), we have $\theta_{N L S}^{*}=\theta_{0}$ and

$$
\begin{aligned}
\sqrt{n}\left(\hat{\theta}_{N L S}-\theta_{0}\right)= & H_{\dot{F}}^{-1} \frac{1}{\sqrt{n}} \sum_{i=1}^{n} \sum_{k=1}^{p}\left\{\epsilon_{i k} \frac{\partial F_{k}\left(t_{i} ; \theta_{0}\right)}{\partial \theta}\right\} \\
& +\sqrt{n} \delta_{n} H_{\dot{F}}^{-1} E\left\{\sum_{k=1}^{p} L_{k}(t) \frac{\partial F_{k}\left(t ; \theta_{0}\right)}{\partial \theta}\right\}+o_{P}(1) .
\end{aligned}
$$

This proposition is essentially a multivariate extension of the nonlinear least squares estimation in the literature (see, e.g. Jennrich (1969); White (1981); Li et al. (2019)).

Using theories of U-statistics, we obtain the following asymptotic properties of $T M_{n}$ under the null and global alternative hypotheses. 
Theorem 1. Given sets $A$ and $B$ of the assumptions in Supplementary Material, if $h \rightarrow 0$ and $n h \rightarrow \infty$, then we have the following.

1. Under the null hypothesis,

$$
T M_{n} \rightarrow \chi_{p}^{2}, \text { in distribution, }
$$

where $\chi_{p}^{2}$ is the chi-square distribution with $p$ degrees of freedom.

2. Under the global alternative $H_{1}$,

$$
T M_{n} /\left(n^{2} h\right) \rightarrow V^{\prime \top} \Sigma^{F \prime-1} V^{\prime}, \text { in probability }
$$

where $V^{\prime}=E\left[\left\{X(t)-F\left(t ; \theta_{1}^{*}\right)\right\}^{2} \odot p(t)\right]$ and $\Sigma^{F^{\prime}}$ is defined as follows:

for any element $\left(k_{1}, k_{2}\right)$ with $1 \leq k_{1}, k_{2} \leq p, \sigma_{k 1 k 2}(t)=E\left(\epsilon_{i k_{1}} \epsilon_{i k_{2}} \mid t\right)$,

$\Sigma_{k_{1} k_{2}}^{F^{\prime}}=2 \int K^{2}(u) d u \int\left[\sigma_{k 1 k 2}(t)+\left\{X_{k_{1}}(t)-F_{k_{1}}\left(t, \theta_{1}^{*}\right)\right\}\left\{X_{k_{2}}(t)-F_{k_{2}}\left(t, \theta_{1}^{*}\right)\right\}\right]^{2} p^{2}(t) d t$.

We see $T M_{n}$ under $H_{1}$ diverges to infinity at a fast rate of order $n^{2} h$.

We also study the asymptotic property of $T M_{n}$ under local alternatives.

Theorem 2. Given sets $A$ and $B$ of the assumptions in Supplementary

Material, if $h \rightarrow 0$ and $n h \rightarrow \infty$, then under $H_{1 n}^{F}$ in (2.7) or $H_{1 n}^{f}$ in (2.8) with $n^{1 / 2} h^{1 / 4} \delta_{n} \rightarrow \infty$,

$$
T M_{n} /\left(n^{2} h \delta_{n}^{4}\right) \rightarrow \mu^{\top} \Sigma^{F-1} \mu \text {, in probability }
$$

where $\mu$ is a p-dimensional vector with the $i$-th component

$$
\mu_{i}=E\left(\left[L_{i}(t)-\frac{\partial F_{i}\left(t ; \theta_{0}\right)}{\partial \theta^{\top}} H_{\dot{F}}^{-1} E\left\{\sum_{k=1}^{p} L_{k}(t) \frac{\partial F_{k}\left(t ; \theta_{0}\right)}{\partial \theta}\right\}\right]^{2} p(t)\right) .
$$


Particularly, if $\delta_{n}=n^{-1 / 2} h^{-1 / 4}$,

$$
T M_{n} \rightarrow \chi_{p}^{2}(\lambda), \text { in distribution }
$$

where $\chi_{p}^{2}(\lambda)$ is noncentral chi-squared distribution where the noncentrality parameter $\lambda=\mu^{\top} \Sigma^{F-1} \mu$ with $\Sigma_{k_{1} k_{2}}^{F}=2 \int K^{2}(u) d u \times \int\left\{\sigma_{k 1 k 2}(t)\right\}^{2} p^{2}(t) d t$.

This result shows that the test can detect the local alternatives distinct from the null at the rate of order $n^{-1 / 2} h^{-1 / 4}$ that is the typical rate local smoothing tests can achieve.

\section{Particularity of checking ODE models}

In the next three sections, we consider the hypotheses in (1.4) for each component. As mentioned before, we cannot use the trajectory matchingbased test for each ODE component. We now give a detailed discussion with three aspects of the particularity of checking ODE models.

1. Mixed components effect. Use a toy example to explain why $T M_{n}$ cannot identify the wrongly modelled component(s). Let the hypothetical model be $\left(X_{1}^{\prime}(t), X_{2}^{\prime}(t)\right)=\left(X_{1}(t), X_{1}(t)+X_{2}(t)\right)$. Yet the true ODE system is $\left(X_{1}^{\prime}(t), X_{2}^{\prime}(t)\right)=\left(2 X_{1}(t), X_{1}(t)+X_{2}(t)\right)$ with the initial values $\left(X_{1}(0), X_{2}(0)\right)=(1,1)$. Here only the first component is wrongly modelled. However, since the second component of $X^{\prime}(t)$ involves both components of 
$X(t)$, the trajectory of the second component in the hypothetical model is $(1+t) \exp (t)$ while in the true system is $\exp (2 t)$. Therefore, if we use a test based on the trajectory of ODE for the second component, the decision of $T M_{n}$ that is based on the second component is strongly disturbed by the model correctness of the first component, and the rightly modelled second component will be rejected. Thus, to construct tests, we should decouple the relationship among different components. We achieve this goal by applying model free nonparametric estimators $\hat{X}(t)$ and $\hat{X}^{\prime}(t)$ later.

2. Mixed parameters effect. If different components share some same parameters, the wrongly modelled component(s) may also make the estimators deviate from the underlying values to have inconsistency. Thus any test relying on these estimators is ineffective. To avoid this problem, we need only use the equation of the tested component to build an objective function for estimation. This can be achieved by using two-step methods. For estimating $\theta_{0 k}$ which consists of the parameters in the $k$-th component, the following gradient matching two-step method is used:

$$
\hat{\theta}_{T S}^{k}=\underset{\theta_{k}}{\operatorname{argmin}} \sum_{j=1}^{m}\left\{\hat{X}_{k}^{\prime}\left(t_{j}^{*}\right)-f_{k}\left(t_{j}^{*}, \hat{X}\left(t_{j}^{*}\right) ; \theta_{k}\right)\right\}^{2} \omega_{k}\left(t_{j}^{*}\right)
$$

with $\omega_{k}(t)$ being a selected weight function and $t_{j}^{*}$ being the selected fitted time grid set by the user (Ding and Wu (2014)). Since only the modelled form of the $k$-th component is used in the objective function, this method 
can avoid the problem of parameters sharing by different components.

In 3.9 , the number $m$ can be larger than $n . \hat{X}(t)$ is the local linear estimator for $X(t)$ and $\hat{X}^{\prime}(t)$ is the local quadratic estimator for $X^{\prime}(t)$ in the vector version, whose $k$-th components $\hat{X}_{k}(t)$ and $\hat{X}_{k}^{\prime}(t)$ are the corresponding local polynomial estimators for $X_{k}(t)$ and $X_{k}^{\prime}(t)$ with the bandwidth $h_{e}$.

3. The choices of different smoothing methods. Different smoothing procedures will be employed in the following tests. How to choose suitable smoothing methods needs careful consideration. If the purpose is to construct a test by generating sample analogue of some quantities at the population level, a simple method such as the Nadaraya-Watson method can be used to make the test simpler in form. However, if the purpose is to give plug-in estimators in the constructed test, we need to reduce the estimation error that would affect the limiting null distribution. In the following, we choose the local linear and quadratic methods to estimate $X(t)$ and $X^{\prime}(t)$, since they have better estimation performances.

\section{Integral matching-based test}

\subsection{Test statistic construction}

Hereafter we omit the indicator $k$ in $\theta_{0 k}, \theta_{k}$ and $\hat{\theta}_{T S}^{k}$ to simplify notation without confusion. Similarly as the integral matching method for estima- 
tion, we construct pseudo-residuals based on the integration representation (1.6):

$$
\hat{e}_{i k}=Y_{i k}-X_{k}\left(t_{0}\right)-\int_{t_{0}}^{t_{i}} f_{k}\left(t, \hat{X}(t) ; \hat{\theta}_{T S}\right) d t .
$$

Here we use the local linear estimator $\hat{X}(t)$ with the bandwidth $h_{0}$ and the two-step estimator $\hat{\theta}_{T S}$. Since $\hat{F}_{k}\left(t_{i} ; \hat{\theta}_{T S}\right)=X_{k}\left(t_{0}\right)+\int_{t_{0}}^{t_{i}} f_{k}\left(t, \hat{X}(t) ; \hat{\theta}_{T S}\right) d t$ is expected to converge to $F_{k}\left(t_{i} ; \theta_{0}\right)$ under the null hypothesis, $\hat{e}_{i k}$ can be used as a surrogate to replace $e_{i k}=Y_{i k}-F_{k}\left(t_{i} ; \hat{\theta}_{N L S}\right)$ in the trajectory matching-based test. This replacement is critical since $\hat{X}(t)$ always capture the true form $X(t)$, which eliminates the influence of latent wrongly modelled components. Note here we require data of all components. Consequently we obtain an integral matching-based test below and write it as $I M_{n}$ in short.

$$
I M_{n}=\sqrt{\frac{n-1}{n}} \frac{n h^{1 / 2} V_{n}^{\hat{F}}}{\sqrt{\widehat{\Sigma} \hat{F}}}=\frac{\sum_{i=1}^{n} \sum_{\substack{j=1 \\ j \neq i}}^{n} K\left(\frac{t_{i}-t_{j}}{h}\right) \hat{e}_{i k} \hat{e}_{j k}}{\left\{\sum_{i=1}^{n} \sum_{\substack{j=1 \\ j \neq i}}^{n} 2 K^{2}\left(\frac{t_{i}-t_{j}}{h}\right) \hat{e}_{i k}^{2} \hat{e}_{j k}^{2}\right\}^{1 / 2}}
$$

where

$$
\begin{aligned}
V_{n}^{\hat{F}} & =\frac{1}{n(n-1)} \sum_{i=1}^{n} \sum_{\substack{j=1 \\
j \neq i}}^{n} \frac{1}{h} K\left(\frac{t_{i}-t_{j}}{h}\right) \hat{e}_{i k} \hat{e}_{j k}, \\
\widehat{\Sigma} \hat{F} & =\frac{2}{n(n-1)} \sum_{i=1}^{n} \sum_{\substack{j=1 \\
j \neq i}}^{n} \frac{1}{h} K^{2}\left(\frac{t_{i}-t_{j}}{h}\right) \hat{e}_{i k}^{2} \hat{e}_{j k}^{2} .
\end{aligned}
$$

\section{$4.2 \quad$ Asymptotic properties}

As in Section 2.2, we suppose that sets A and B of the assumptions in Sup- 
plementary Material hold. Note that assumptions (3) and (4) in set A are useful to ensure the uniform convergence rate for nonparametric estimation (Hansen (2008)). We also suppose the assumptions in set $\mathrm{C}$ hold. These assumptions are similar to those in Liang and $\mathrm{Wu}$ (2008), containing the conditions on $f(t, X(t) ; \theta)$ that are necessary for the two-step method.

In the following, we also consider two local alternatives that correspond to 2.7 and $(2.8)$ for any component function:

$$
H_{1 k n}^{F}: X_{k}(t)=F_{k}\left(t ; \theta_{0}\right)+\delta_{n} L_{k}(t)
$$

with $X_{k}(t) \neq F_{k}(t ; \theta)$ for every $\theta \in \Theta$, and

$$
H_{1 k n}^{f}: X_{k}^{\prime}(t)=f_{k}\left(t, X(t) ; \theta_{0}\right)+\delta_{n} l_{k}(t)
$$

with $X_{k}^{\prime}(t) \neq f_{k}(t, X(t) ; \theta)$ for every $\theta \in \Theta$. Here the subscript $k$ represents the $k$-th component. Using Proposition 1, we can define the counterpart functions $l_{k}(t)$ under $H_{1 k n}^{F}$ and $L_{k}(t)$ under $H_{1 k n}^{f}$, then uniformly deal with these two kinds of local alternatives.

We first give the asymptotic properties of $\hat{\theta}_{T S}$ defined in 3.9 under different hypotheses, which are not available in the literature. Assumption (3) in Set $\mathrm{C}$ ensures that there exists a unique minimizer $\theta_{T S}^{*}=$ $\arg \min _{\theta} E_{p^{*}}\left[\left\{X_{k}^{\prime}(t)-f_{k}(t, X(t), \theta)\right\}^{2} w_{k}(t)\right]$, where $p^{*}(t)$ is the probability density function of the selected fitted time point. Denote $\Lambda(t)=$ 
$\hat{X}(t)-X(t), \Delta(t)=\hat{X}^{\prime}(t)-X^{\prime}(t)$. We have the following.

Proposition 3. Given sets $A$ and $C$ of the assumptions in Supplementary Material, $\log n /\left(n h_{e}^{3}\right)=o(1)$, the two-step estimator $\hat{\theta}_{T S}$ is consistent to $\theta_{T S}^{*}$ and has the following asymptotic representations.

1. Under the null hypothesis, we have $\theta_{T S}^{*}=\theta_{0}$ and letting

$$
H_{\dot{f}}=E_{p^{*}}\left\{\omega_{k}(t) \frac{\partial f_{k}\left(t, X(t) ; \theta_{0}\right)}{\partial \theta} \frac{\partial f_{k}\left(t, X(t) ; \theta_{0}\right)}{\partial \theta^{\top}}\right\}
$$

then

$$
\begin{aligned}
\hat{\theta}_{T S}-\theta_{0}= & H_{\dot{f}}^{-1} \frac{1}{m} \sum_{j=1}^{m}\left\{\Delta_{k}\left(t_{j}^{*}\right) \omega_{k}\left(t_{j}^{*}\right) \frac{\partial f_{k}\left(t_{j}^{*}, X\left(t_{j}^{*}\right) ; \theta_{0}\right)}{\partial \theta}\right. \\
& \left.-\omega_{k}\left(t_{j}^{*}\right) \frac{\partial f_{k}\left(t_{j}^{*}, X\left(t_{j}^{*}\right) ; \theta_{0}\right)}{\partial \theta} \frac{\partial f_{k}\left(t_{j}^{*}, X\left(t_{j}^{*}\right) ; \theta_{0}\right)}{\partial X^{\top}} \Lambda\left(t_{j}^{*}\right)\right\}+o_{P}\left(n^{-1 / 2}\right)
\end{aligned}
$$

which is a term of order $O_{P}\left(n^{-1 / 2}\right)$.

2. Under the global alternative hypothesis $H_{1}$, we have $\theta_{T S}^{*}=\theta_{1}$ and

$$
\begin{aligned}
& \sqrt{n}\left(\hat{\theta}_{T S}-\theta_{1}\right) \\
= & G^{-1} \frac{\sqrt{n}}{m} \sum_{j=1}^{m}\left\{\Delta_{k}\left(t_{j}^{*}\right) \omega_{k}\left(t_{j}^{*}\right) \frac{\partial f_{k}\left(t_{j}^{*}, X\left(t_{j}^{*}\right) ; \theta_{1}\right)}{\partial \theta}\right. \\
& \left.-\omega_{k}\left(t_{j}^{*}\right) \frac{\partial f_{k}\left(t_{j}^{*}, X\left(t_{j}^{*}\right) ; \theta_{1}\right)}{\partial \theta} \frac{\partial f_{k}\left(t_{j}^{*}, X\left(t_{j}^{*}\right) ; \theta_{1}\right)}{\partial X^{\top}} \Lambda\left(t_{j}^{*}\right)\right\}+o_{P}(1)
\end{aligned}
$$

where

$$
\begin{aligned}
G= & E_{p^{*}}\left\{\omega_{k}(t) \frac{\partial f_{k}\left(t, X(t) ; \theta_{1}\right)}{\partial \theta} \frac{\partial f_{k}\left(t, X(t) ; \theta_{1}\right)}{\partial \theta^{\top}}\right\} \\
& -E_{p^{*}}\left[\left\{X_{k}(t)-f_{k}\left(t, X(t) ; \theta_{1}\right)\right\} \omega_{k}(t) \frac{\partial^{2} f_{k}\left(t, X(t) ; \theta_{1}\right)}{\partial \theta \partial \theta^{\top}}\right] .
\end{aligned}
$$


3. Under the local alternative hypothesis $H_{1 k n}^{F}$ in (4.10) or $H_{1 k n}^{f}$ in (4.11) with $\delta_{n} \rightarrow 0$, we have $\theta_{T S}^{*}=\theta_{0}$ and

$$
\begin{aligned}
\sqrt{n}\left(\hat{\theta}_{T S}-\theta_{0}\right)= & H_{\dot{f}}^{-1} \frac{\sqrt{n}}{m} \sum_{j=1}^{m}\left\{\Delta_{k}\left(t_{j}^{*}\right) \omega_{k}\left(t_{j}^{*}\right) \frac{\partial f_{k}\left(t_{j}^{*}, X\left(t_{j}^{*}\right) ; \theta_{0}\right)}{\partial \theta}\right. \\
& \left.-\omega_{k}\left(t_{j}^{*}\right) \frac{\partial f_{k}\left(t_{j}^{*}, X\left(t_{j}^{*}\right) ; \theta_{0}\right)}{\partial \theta} \frac{\partial f_{k}\left(t_{j}^{*}, X\left(t_{j}^{*}\right) ; \theta_{0}\right)}{\partial X^{\top}} \Lambda\left(t_{j}^{*}\right)\right\} \\
& +\sqrt{n} \delta_{n} H_{\dot{f}}^{-1} E_{p^{*}}\left\{l(t) \omega(t) \frac{\partial f_{k}\left(t, X(t) ; \theta_{0}\right)}{\partial \theta}\right\}+o_{P}(1) .
\end{aligned}
$$

Having Proposition 3, we turn to study the asymptotic properties of $I M_{n}$. Let $\varepsilon_{i k}=Y_{i k}-X_{k}\left(t_{0}\right)-\int_{t_{0}}^{t_{i}} f_{k}\left(t, X(t) ; \theta_{T S}^{*}\right) d t$ be the residual. By denoting $\hat{e}_{i k}=\hat{e}_{i k}+\varepsilon_{i k}-\varepsilon_{i k}, V_{n}^{\hat{F}}$ is an asymptotic U-statistic. To handle this U-statistic, we give a proposition about a non-degenerate U-statistic of order $m^{*}$ with a kernel varying with $n$.

Proposition 4. Suppose $U_{n}$ is a non-degenerate $U$-statistic with the kernel $h_{n}\left(z_{1}, \cdots, z_{m^{*}}\right)$ of order $m^{*}$. If $E\left[\left\|h_{n}\left(z_{1}, \cdots, z_{m^{*}}\right)\right\|^{2}\right]=o(n)$, then

$$
\sqrt{n}\left(U_{n}-\hat{U}_{n}\right)=o_{P}(1)
$$

where

$\hat{U}_{n}=E\left\{h_{n}\left(z_{1}, \cdots, z_{m^{*}}\right)\right\}+\frac{m^{*}}{n} \sum_{i=1}^{n}\left\{E\left[h_{n}\left(z_{1}, \cdots, z_{m^{*}}\right) \mid z_{i}\right]-E\left[h_{n}\left(z_{1}, \cdots, z_{m^{*}}\right)\right]\right\}$ is the projection of $U_{n}$.

Applying Proposition 4, it can be proven the replacement of $\hat{e}_{i k}$ to $e_{i k}$ have no influence to the limiting null distribution of $I M_{n}$. By using 
this property, we state the asymptotic properties of $I M_{n}$ in the following theorem. Recall that $\hat{X}(t)$ is the local linear estimator of $X(t)$ with the bandwidth $h_{0}$. Define $a_{n}\left(h_{0}\right)=h_{0}^{2}+n^{-1 / 2} h_{0}^{-1 / 2} \log n^{1 / 2}$ which is the uniform convergence rate of local linear estimator (Hansen (2008)).

Theorem 3. Given sets $A-C$ of the assumptions in Supplementary Material, if $h \rightarrow 0, n h \rightarrow \infty, n^{-1 / 2} h^{-1 / 2}=o\left(h_{0}\right)$ and $a_{n}^{2}\left(h_{0}\right)=o\left(n^{-1} h^{-1 / 2}\right)$, then we have the following.

1. Under the null hypothesis,

$$
I M_{n} \rightarrow N(0,1) \text {, in distribution }
$$

2. Under the global alternative, $I M_{n} /\left(n h^{1 / 2}\right) \rightarrow I M_{H 1}$ in probability, where

$$
I M_{H 1}=\frac{E\left[\left\{X_{k}(t)-F_{k}^{*}\left(t ; \theta_{1}\right)\right\}^{2} p(t)\right]}{\left(2 \int K^{2}(u) d u \int\left[\sigma_{k}^{2}(t)+\left\{X_{k}(t)-F_{k}^{*}\left(t ; \theta_{1}\right)\right\}^{2}\right]^{2} p^{2}(t) d t\right)^{1 / 2}},
$$

with $\quad F_{k}^{*}\left(t ; \theta_{1}\right)=X_{k}\left(t_{0}\right)+\int_{t_{0}}^{t} f_{k}\left(t, X(s) ; \theta_{1}\right) d s \quad$ and $\quad \sigma_{k}(t)=E\left(\epsilon_{i k}^{2} \mid t\right)$.

Theorem 3 shows that this test is consistent and it diverges to infinity at the rate of order $n h^{1 / 2}$ under $H_{1 k}$. The following theorem states the asymptotic property of $I M_{n}$ under the local alternatives.

Theorem 4. Assume the conditions in Theorem 3 with $n^{1 / 2} h^{1 / 4} \delta_{n} \rightarrow \infty$. Then we have, under $H_{1 k n}^{F}$ in (4.10) or $H_{1 k n}^{f}$ in 4.11,

$$
I M_{n} /\left(n h^{1 / 2} \delta_{n}^{2}\right) \rightarrow \mu_{I} / \sigma_{k}^{*}, \text { in probability }
$$


where

$$
\begin{aligned}
\mu_{I}= & E\left(\left[L_{k}(t)-\frac{\partial F_{k}\left(t ; \theta_{0}\right)}{\partial \theta^{\top}} H_{\dot{f}}^{-1} E_{p^{*}}\left\{l_{k}(t) \omega_{k}(t) \frac{\partial f_{k}\left(t, X(t) ; \theta_{0}\right)}{\partial \theta}\right\}\right]^{2} p(t)\right) \\
& +2 E\left(\left[\frac{\partial F_{k}\left(t ; \theta_{0}\right)}{\partial \theta^{\top}} H_{\dot{f}}^{-1} E_{p^{*}}\left\{l_{k}(t) \omega_{k}(t) \frac{\partial f_{k}\left(t, X(t) ; \theta_{0}\right)}{\partial \theta}\right\}-L_{k}(t)\right] p(t)\right. \\
& \left.\times \int_{t_{0}}^{t} \frac{\partial f_{k}\left(s, X(s) ; \theta_{0}\right)}{\partial X^{T}} L(s) d s\right)+E\left[\left\{\int_{t_{0}}^{t} \frac{\partial f_{k}\left(s, X(s) ; \theta_{0}\right)}{\partial X^{T}} L(s) d s\right\}^{2} p(t)\right], \\
\sigma_{k}^{*}= & {\left[2 \int K^{2}(u) d u \int\left\{\sigma_{k}^{2}(t)\right\}^{2} p^{2}(t) d t\right]^{1 / 2} . }
\end{aligned}
$$

Particularly, when $\delta_{n}=n^{-1 / 2} h^{-1 / 4}$,

$$
I M_{n} \rightarrow N\left(\mu_{I} / \sigma_{k}^{*}, 1\right), \text { in distribution }
$$

\section{Gradient matching-based test}

\subsection{Test statistic construction}

As we note in the integral matching-based test, the surrogate $\hat{F}_{k}\left(t_{i} ; \hat{\theta}_{T S}\right)=$ $X_{k}\left(t_{0}\right)+\int_{t_{0}}^{t_{i}} f_{k}\left(t, \hat{X}(t) ; \hat{\theta}_{T S}\right) d t$ involves both the estimation of $X(t)$ and the integral over the function $f_{k}(\cdot)$. In other words, the cumulative estimation error could be large in finite sample scenarios. Some numerical studies also suggested this problem. Thus, we in this section consider a test that is directly based on the gradient representation (1.1). With $n^{*} \leq n$ observations, we give the Nadaraya-Watson kernel estimator of $X^{\prime}(t)$ as,

$$
\tilde{X}^{\prime}(t)=\frac{\hat{\psi}^{\prime}(t) \hat{p}(t)-\hat{\psi}(t) \hat{p}^{\prime}(t)}{\hat{p}^{2}(t)}
$$


where

$$
\begin{aligned}
& \hat{\psi}(t)=\frac{1}{n^{*}} \sum_{i=1}^{n^{*}} \frac{1}{h} K\left(\frac{t-t_{i}}{h}\right) Y_{i} \quad \hat{\psi}^{\prime}(t)=\frac{1}{n^{*}} \sum_{i=1}^{n^{*}} \frac{1}{h^{2}} K^{\prime}\left(\frac{t-t_{i}}{h}\right) Y_{i} \\
& \hat{p}(t)=\frac{1}{n^{*}} \sum_{i=1}^{n^{*}} \frac{1}{h} K\left(\frac{t-t_{i}}{h}\right) \quad \hat{p}^{\prime}(t)=\frac{1}{n^{*}} \sum_{i=1}^{n^{*}} \frac{1}{h^{2}} K^{\prime}\left(\frac{t-t_{i}}{h}\right) .
\end{aligned}
$$

Note that $e_{f}(t)=X_{k}^{\prime}(t)-f_{k}\left(t, X(t) ; \theta_{0}\right)=0$ corresponds to the null hypothesis, otherwise to the alternative hypothesis. Thus, if we replace $X_{k}^{\prime}(t)$ by $\tilde{X}_{k}^{\prime}(t)$ and $f_{k}\left(t, X(t) ; \theta_{0}\right)$ by $f_{k}\left(t, \hat{X}(t) ; \hat{\theta}_{T S}\right)$ with $\hat{\theta}_{T S}$ defined in 3.9$)$, the pseudo-residual $\hat{e}_{f}(t)=\tilde{X}_{k}^{\prime}(t)-f_{k}\left(t, \hat{X}(t) ; \hat{\theta}_{T S}\right)$ is expected to converge to zero in probability. Then, $E\left\{\hat{e}_{f}^{2}\left(t_{i}\right) \hat{p}^{4}\left(t_{i}\right)\right\}$ is expected to converge to zero under the null while to a positive constant under the alternatives, where $\hat{p}^{4}\left(t_{i}\right)$ is used to eliminate the denominator in the nonparametric estimation. Therefore, its empirical version seems reasonably a test statistic:

$$
\begin{aligned}
V_{n^{*}}^{f}= & \frac{1}{n^{*} h^{2}} \sum_{d=1}^{n^{*}}\left\{\tilde{X}_{k}^{\prime}\left(t_{d}\right)-f_{k}\left(t, \hat{X}\left(t_{d}\right) ; \hat{\theta}_{T S}\right)\right\}^{2} \hat{p}^{4}\left(t_{d}\right) \\
= & \frac{1}{n^{*} h^{2}} \sum_{d=1}^{n^{*}}\left[\frac { 1 } { n ^ { 2 } } \sum _ { i = 1 } ^ { n ^ { * } } \sum _ { j = 1 } ^ { n ^ { * } } \left\{\frac{1}{h^{3}} K^{\prime}\left(\frac{t_{d}-t_{i}}{h}\right) K\left(\frac{t_{d}-t_{j}}{h}\right)\left(Y_{i k}-Y_{j k}\right)\right.\right. \\
& \left.\left.-\frac{1}{h^{2}} K\left(\frac{t_{d}-t_{i}}{h}\right) K\left(\frac{t_{d}-t_{j}}{h}\right) f_{k}\left(t, \hat{X}\left(t_{d}\right) ; \hat{\theta}_{T S}\right)\right\}\right]^{2} .
\end{aligned}
$$

$1 / h^{2}$ is added to obtain a non-degenerate limit, since $\hat{e}_{f}(t)$ converges to 0 rather than a zero mean random variable in probability under the null.

However, as the nonparametric kernel estimation is biased, when we choose $n^{*}=n, V_{n}^{f}$ has a non-negligible bias even under $H_{0}$. This bias is 
hard to estimate which causes the difficulty to analyze the limiting null distribution. Thus we suggest a data splitting method to build a new test.

To this end, we randomly partition the original sample into 2 subsamples $n^{*}=\tilde{n}$ and $n^{*}=n-\tilde{n}$ where $\tilde{n}=\lfloor n / 2\rfloor$. Using these two subsamples, we construct two statistics $V_{\tilde{n} 1}^{f}$ and $V_{(n-\tilde{n}) 2}^{f}$. As $n-2 \tilde{n} \leq 1$, the asymptotic properties of $V_{(n-\tilde{n}) 2}^{f}$ should be the same as those of $V_{\tilde{n} 2}^{f}$. Thus we assume that, without loss of generality, $n=2 \tilde{n}$ is even. Therefore, the difference $V_{\tilde{n} 1}^{f}-V_{\tilde{n} 2}^{f}$ can serve as a statistic which is symmetric about zero to determine the limiting null distribution. To make the test have power, we utilize another statistic $\hat{S}=1 / h^{2} \int\left\{f_{k}\left(t, \hat{X}(t) ; \hat{\theta}_{T S}\right)-\hat{X}_{k}^{\prime}(t)\right\}^{2} d t$ which is an estimator of $S=1 / h^{2} \int\left\{f_{k}(t, X(t) ; \theta)-X_{k}^{\prime}(t)\right\}^{2} d t$ that is equal to zero under the null and larger than zero under the alternatives. The new test statistic is their convex combination: $V_{\tilde{n} 1}^{f}-V_{\tilde{n} 2}^{f}+c \hat{S}$. The key point is that, by the proper choice of the bandwidth parameters, $\hat{S}$ has faster rate of convergence to zero than $V_{\tilde{n} 1}^{f}-V_{\tilde{n} 2}^{f}$ under the null hypothesis. It then does not change the limiting distribution under the null hypothesis, while provides power under the alternatives. The constant $c$ is a tuning parameter set by user.

By dividing by the estimator of its variance, the final test statistic is

$$
G M_{n}=\frac{\sqrt{\tilde{n}}\left(V_{\tilde{n} 1}^{f}-V_{\tilde{n} 2}^{f}+c \hat{S}\right)}{\sqrt{2 \widehat{\Sigma}^{f}}},
$$


where

$$
\begin{gathered}
\widehat{\Sigma}^{f}=\frac{1}{n-1} \sum_{s=1}^{n}\left\{\hat{w}_{n}\left(z_{s}\right)-\frac{1}{n} \sum_{i=1}^{n} \hat{w}_{n}\left(z_{i}\right)\right\}^{2}, \\
\hat{w}_{n}\left(z_{s}\right)=\frac{1}{\left\lfloor\frac{n-1}{4}\right\rfloor} \sum_{i=1}^{\left\lfloor\frac{n-1}{4}\right\rfloor} W_{n}\left(z_{i_{1}}, z_{i_{2}}, z_{i_{3}}, z_{i_{4}}, z_{s}\right), \\
W_{n}\left(z_{a}, z_{b}, z_{c}, z_{d}, z_{s}\right)=\frac{1}{5 !} \sum_{P} W_{n}^{\prime}\left(z_{i_{1}}, z_{i_{2}}, z_{i_{3}}, z_{i_{4}}, z_{i_{5}}\right) \\
W_{n}^{\prime}\left(z_{a}, z_{b}, z_{c}, z_{d}, z_{s}\right)=\frac{1}{h^{2}} K\left(\frac{t_{s}-t_{a}}{h}\right) K\left(\frac{t_{s}-t_{b}}{h}\right) \\
\times\left\{\frac{1}{h^{3}} K^{\prime}\left(\frac{t_{s}-t_{c}}{h}\right)\left(Y_{c k}-Y_{a k}\right)-\frac{1}{h^{2}} K\left(\frac{t_{s}-t_{c}}{h}\right) f_{k}\left(t_{s}, \hat{X}\left(t_{s}\right) ; \hat{\theta}_{T S}\right)\right\} \\
\times\left\{\frac{1}{h^{3}} K^{\prime}\left(\frac{t_{s}-t_{d}}{h}\right)\left(Y_{d k}-Y_{b k}\right)-\frac{1}{h^{2}} K\left(\frac{t_{s}-t_{d}}{h}\right) f_{k}\left(t_{s}, \hat{X}\left(t_{s}\right) ; \hat{\theta}_{T S}\right)\right\} .
\end{gathered}
$$

Here $W_{n}(\cdot)$ is the symmetric version of $W_{n}^{\prime}(\cdot)$ and $\sum_{P}$ means that the sum is taken over all permutations $\left(i_{1}, i_{2}, i_{3}, i_{4}, i_{5}\right)$ of $\{a, b, c, d, s\}$. The local linear and local quadratic smoother are used to obtain $\hat{X}(t)$ and $\hat{X}^{\prime}(t)$ respectively with the corresponding bandwidths $h_{0}$ and $h_{1}$.

\subsection{Asymptotic properties}

To derive the properties, we in this Section give sets $\mathrm{A}$ and $\mathrm{C}$ of the assumptions in Supplementary Material. Let $b_{n}(h)=h^{2}+n^{-1 / 2} h^{-3 / 2} \log n$ which is the uniform convergence rate of $X^{\prime}(t)($ Liang and $\mathrm{Wu}(2008))$. We state asymptotic properties of $G M_{n}$ under the null and global alternative hypotheses. 
Theorem 5. Given sets $A$ and $C$ of the assumptions in Supplementary Material, if $h^{-12}=o(n), a_{n}^{2}\left(h_{0}\right) h^{-2}=o\left(n^{-1 / 2}\right)$ and $b_{n}^{2}\left(h_{1}\right) h^{-2}=o\left(n^{-1 / 2}\right)$, recalling that $\tilde{n}=\lfloor n / 2\rfloor$, we have the following.

1. Under the null hypothesis,

$$
G M_{n} \rightarrow N(0,1), \text { in distribution }
$$

2. Under the global alternative,

$$
G M_{n} / \sqrt{\tilde{n}} \rightarrow \frac{c \int\left[f_{k}\left(t, X(t) ; \theta_{1}\right)-X_{k}^{\prime}(t)\right]^{2} d t}{\sqrt{2 \Sigma^{f^{\prime}}}}>0 \text {, in probability }
$$

where

$$
\begin{aligned}
\Sigma^{f \prime}= & \int\left[25\left\{f_{k}\left(t, X(t) ; \theta_{1}\right)-X_{k}^{\prime}(t)\right\}^{4} p^{8}(t)+4\left\{f_{k}^{\prime}\left(t, X(t) ; \theta_{1}\right)-X_{k}^{(2)}(t)\right\}^{2} \sigma_{k}^{2}(t) p^{8}(t)\right] d t \\
& -25\left[\int\left\{f_{k}\left(t, X(t) ; \theta_{1}\right)-X_{k}^{\prime}(t)\right\}^{2} p^{4}(t) d t\right]^{2} .
\end{aligned}
$$

Theorem 5 shows that the test is consistent and diverges to infinity at the rate of $\sqrt{n}$ under global alternatives although, like local smoothing tests, nonparametric technique is used. The following theorem states the asymptotic power of $G M_{n}$ under $H_{1 k n}^{F}$ and $H_{1 k n}^{f}$.

Theorem 6. Assume the conditions in Theorem 5 hold, then under $H_{1 k n}^{F}$ in (4.10) or $H_{1 k n}^{f}$ in 4.11), with $\tilde{n}^{1 / 4} h^{-1} \delta_{n} \rightarrow \infty$ and $\delta_{n} h^{-1}=o(1)$,

$$
G M_{n} /\left(\tilde{n}^{1 / 2} h^{-2} \delta_{n}^{2}\right) \rightarrow c \mu_{G} / \sqrt{2 \Sigma^{f}}, \text { in probability }
$$


where

$$
\begin{aligned}
\mu_{G}= & {\left[H_{\dot{f}}^{-1} E_{p^{*}}\left\{l_{k}(t) \omega_{k}(t) \frac{\partial f_{k}\left(t, X(t) ; \theta_{0}\right)}{\partial \theta}\right\}\right]^{\top} \times\left\{\int \frac{\partial f_{k}\left(t, X(t) ; \theta_{0}\right)}{\partial \theta} \frac{\partial f_{k}\left(t, X(t) ; \theta_{0}\right)}{\partial \theta^{\top}} d t\right\} } \\
& \times\left[H_{\dot{f}}^{-1} E_{p^{*}}\left\{l_{k}(t) \omega_{k}(t) \frac{\partial f_{k}\left(t, X(t) ; \theta_{0}\right)}{\partial \theta}\right\}\right]+\int l_{k}^{2}(t) d t \\
& -2\left\{\int l_{k}(t) \frac{\partial f_{k}\left(t, X(t) ; \theta_{0}\right)}{\partial \theta^{\top}} d t\right\} H_{\dot{f}}^{-1} E_{p^{*}}\left\{l_{k}(t) \omega_{k}(t) \frac{\partial f_{k}\left(t, X(t) ; \theta_{0}\right)}{\partial \theta}\right\}, \\
\Sigma^{f}= & \frac{1}{9}\left\{\int u^{3} K^{\prime}(u) d u\right\}^{2} \int\left\{X_{k}^{(4)}(t)\right\}^{2} \sigma_{k}^{2}(t) p^{8}(t) d t .
\end{aligned}
$$

Particularly, if $\delta_{n}=\tilde{n}^{-1 / 4} h$,

$$
G M_{n} \rightarrow N\left(c \mu_{G} / \sqrt{2 \Sigma^{f}}, 1\right) \text {, in distribution. }
$$

\section{Numerical studies}

\subsection{Simulations}

We conduct four simulation studies to evidence the performances of the proposed tests in finite sample scenarios. In Examples 1-3, we use $T M_{n}$ to check the whole ODE system while use $I M_{n}$ and $G M_{n}$ to check each component. The subscript is used to denote which component the tests check. For example, $G M_{n 1}$ means the $G M_{n}$ test for the first component. In Example 4, we reconsider models in Examples 1-3 as partially observed ODE systems. Suppose that only data of the second component are measured and use $T M_{n}^{o}$ to check these models. As a competitor with $T M_{n}$, we also apply Hooker (2009)'s test $T^{H}$ to check the whole ODE system. 
In particular, the simulation results show that the empirical size of $I M_{n}$ is often very large in the complex ODE model settings. This may be because the nonparametric estimation $\hat{X}(t)$ for all time points $t$ have estimation error, and the integral over the surrogate $\hat{e}_{i k}$ of $e_{i k}$ in finite sample scenarios could cause very large cumulative error of $I M_{n}$. Thus, to control the empirical size of $I M_{n}$, we use an adjusted version in simulation.

First, we restrict the integral in the shorter interval $(0.1,0.9)$ rather than the whole time interval $(0,1)$ to avoid the boundary effect. Second, to reduce the error caused by the integration, we split the interval into $n_{l}=8$ equidistant parts $\mathcal{T}_{l}=(l / 10,(l+1) / 10), l=1,2, \cdots, 8$ and define

$$
\begin{aligned}
\hat{e}_{i k}^{l} & =\sum_{l=1}^{8}\left\{Y_{i k}-\hat{X}_{k}\left(\frac{l}{10}\right)-\int_{\min \left(\frac{l}{10}, t_{i}\right)}^{t_{i}} f_{k}\left(t, \hat{X}(t) ; \hat{\theta}_{T S}\right) d t\right\} I\left(t_{i} \in \mathcal{T}_{l}\right), \\
I M_{n}^{l} & =\frac{\sum_{i=1}^{n} \sum_{\substack{j=1 \\
j \neq i}}^{n} K\left(\frac{t_{i}-t_{j}}{h}\right) \hat{e}_{i k}^{l} \hat{e}_{j k}^{l}}{\left\{\sum_{i=1}^{n} \sum_{\substack{j=1 \\
j \neq i}}^{n} 2 K^{2}\left(\frac{t_{i}-t_{j}}{h}\right) \hat{e}_{i k}^{l 2} \hat{e}_{j k}^{l 2}\right\}^{1 / 2}},
\end{aligned}
$$

where $I(\cdot)$ is the characteristic function. Then we define a test statistic as

$$
I M_{n}^{*}=\frac{\sum_{l=1}^{8} I M_{n}^{l}}{2 \sqrt{2} \nu_{n}}
$$

where $\nu_{n}=1+2 n^{-1 / 2}$ is used to further reduce the magnitude. It can be shown this test has the same asymptotic normality as the original one under the null by using Cramér-Wald device and continuous mapping theorem. About the selection of smoothing parameters and other simulation settings, 
see Supplementary Material for details.

Example 1. Data sets are generated from the following ODE models:

$$
\begin{array}{cc}
H_{11}: & X^{\prime}(t)=\left[\begin{array}{c}
\frac{d X_{1}}{d t} \\
\frac{d X_{2}}{d t}
\end{array}\right]=\tau\left[\begin{array}{c}
a X_{1}+0.4 \alpha \cos \left(a X_{1}\right) \\
a X_{1}+b X_{2}+0.4 \beta \cos \left(a X_{1}+b X_{2}\right)
\end{array}\right], \\
H_{12}: & X^{\prime}(t)=\left[\begin{array}{c}
\frac{d X_{1}}{d t} \\
\frac{d X_{2}}{d t}
\end{array}\right]=\tau\left[\begin{array}{c}
a X_{1}+0.1 \alpha\left(a X_{1}\right)^{3} \\
a X_{1}+b X_{2}+0.1 \beta\left(a X_{1}+b X_{2}\right)^{3}
\end{array}\right], \\
H_{13}: & X^{\prime}(t)=\left[\begin{array}{c}
a X_{1} \\
d t \\
\frac{d X_{2}}{d t}
\end{array}\right]=\tau\left[\begin{array}{c}
a \alpha \exp \left(a X_{1}\right) \\
a X_{1}+b X_{2}+5 \beta \exp \left(a X_{1}+b X_{2}\right)
\end{array}\right] .
\end{array}
$$

We consider three cases in which the linear null ODE models are added with different disturbance terms to form alternative ODE models. The alternatives are oscillating functions of $X$ in $H_{11}$ while they are low-frequent functions of $X$ in both $H_{12}$ and $H_{13}$. In each case, $\alpha=0$ and $\beta=0$ correspond to the null, otherwise to the alternative hypothesis. When only one of $\alpha$ and $\beta$ is nonzero, only one element ODE function is different under the alternative hypothesis. When $\alpha$ and $\beta$ are both nonzero, both components are then changed. $\tau$ is a timescale parameter that transforms the arbitrary length of time interval to 1 . We set the true parameter $(a, b)=$ $(-0.06,-0.24), \tau=10, \sigma_{\epsilon}=0.05$ and the sample size is 300 . The empirical sizes and powers with a 0.05 significance level are presented in Table 1. 
The results show that $T M_{n}$ maintains the significance level. It also has very good powers under all of the alternative models, which are significantly larger than $I M_{n}$ and $G M_{n}$. This is not surprising because $T M$ summarizes the deviation of all the components from the trajectory of the null model. $T^{H}$ also maintains the significance level well with good powers.

Recall the subscript represents the component to be checked. By and large, $I M_{n 1}$ and $I M_{n 2}$ can maintain the significance level, although in some cases the empirical sizes of $I M_{n 1}$ are slightly lower than the significance level. $I M_{n 1}$ and $I M_{n 2}$ have high powers in most cases. $G M_{n 1}$ and $G M_{n 2}$ can basically maintain the significance level. $G M_{n 1}$ has good powers in all three cases while the powers of $G M_{n 2}$ vary in different cases. In the last two cases, when $(\alpha, \beta)=(1,1), G M_{n 2}$ has low powers $(0.600,0.095)$, while when $(\alpha, \beta)=(0,1)$, it has higher powers $(0.734,0.120)$. This phenomenon is worthwhile to pay attention and very different from classical testing for regressions. A possible explanation would be that an extra $\alpha$ suppresses the influence of $\beta$ term, making the disturbance term in $\hat{X}^{\prime}(t)$ less important.

In the third case, $G M_{n 1}$ shows greater powers than $I M_{n 1}$. However, considering $G M_{n 2}$ and $I M_{n 2}$, the situation is just on the contrary. Since $I M_{n}$ and $G M_{n}$ measure different deviation indices and have diverse normalizing factors, they show distinct sensitivity superiority in different settings. 
Table 1: Empirical sizes and powers in Example 1.

\begin{tabular}{|c|c|c|c|c|c|c|c|c|}
\hline Hypothesis & $\alpha$ & $\beta$ & $T M_{n}$ & $I M_{n 1}$ & $I M_{n 2}$ & $G M_{n 1}$ & $G M_{n 2}$ & $T^{H}$ \\
\hline \multirow[t]{7}{*}{$H_{11}$} & 0 & 0 & 0.045 & 0.036 & 0.058 & 0.038 & 0.050 & 0.047 \\
\hline & 0.5 & 0 & 1.000 & 0.510 & 0.059 & 0.279 & 0.034 & 1.000 \\
\hline & 0 & 0.5 & 1.000 & 0.030 & 0.999 & 0.042 & 0.191 & 1.000 \\
\hline & 0.5 & 0.5 & 1.000 & 0.499 & 0.998 & 0.270 & 0.193 & 1.000 \\
\hline & 1 & 0 & 1.000 & 1.000 & 0.059 & 1.000 & 0.044 & 1.000 \\
\hline & 0 & 1 & 1.000 & 0.021 & 1.000 & 0.035 & 0.994 & 1.000 \\
\hline & 1 & 1 & 1.000 & 0.997 & 1.000 & 1.000 & 0.994 & 1.000 \\
\hline \multirow[t]{7}{*}{$H_{12}$} & 0 & 0 & 0.043 & 0.023 & 0.047 & 0.035 & 0.048 & 0.049 \\
\hline & 0.5 & 0 & 1.000 & 1.000 & 0.052 & 0.915 & 0.036 & 1.000 \\
\hline & 0 & 0.5 & 1.000 & 0.026 & 1.000 & 0.039 & 0.661 & 1.000 \\
\hline & 0.5 & 0.5 & 1.000 & 1.000 & 1.000 & 0.919 & 0.606 & 1.000 \\
\hline & 1 & 0 & 1.000 & 1.000 & 0.051 & 0.998 & 0.045 & 1.000 \\
\hline & 0 & 1 & 1.000 & 0.026 & 0.998 & 0.031 & 0.734 & 1.000 \\
\hline & 1 & 1 & 1.000 & 1.000 & 0.993 & 0.997 & 0.600 & 1.000 \\
\hline \multirow[t]{7}{*}{$H_{13}$} & 0 & 0 & 0.046 & 0.026 & 0.049 & 0.037 & 0.050 & 0.059 \\
\hline & 0.5 & 0 & 1.000 & 0.126 & 0.037 & 0.294 & 0.034 & 1.000 \\
\hline & 0 & 0.5 & 1.000 & 0.033 & 0.849 & 0.040 & 0.078 & 1.000 \\
\hline & 0.5 & 0.5 & 1.000 & 0.134 & 0.721 & 0.296 & 0.062 & 1.000 \\
\hline & 1 & 0 & 1.000 & 0.171 & 0.044 & 0.906 & 0.048 & 1.000 \\
\hline & 0 & 1 & 1.000 & 0.025 & 0.982 & 0.031 & 0.120 & 1.000 \\
\hline & 1 & 1 & 1.000 & 0.161 & 0.875 & 0.893 & 0.095 & 1.000 \\
\hline
\end{tabular}


Example 2. Data sets are generated from the following ODE models:

$$
H_{2}: \quad X^{\prime}(t)=\left[\begin{array}{c}
\frac{d X_{1}}{d t} \\
\frac{d X_{2}}{d t}
\end{array}\right]=\tau\left[\begin{array}{c}
a\left(X_{1}+X_{2}-\frac{X_{1}^{3}}{3}\right)+\alpha X_{1} X_{2} \\
-\frac{X_{1}+b X_{2}-c}{a}+0.4 \beta X_{1} X_{2}
\end{array}\right] .
$$

This is the famous FitzHugh-Nagumo ODE system which describes the behavior of spike potentials in the giant axon of squid neurons (FitzHugh (1961); Nagumo et al. (1962)). Following Ding and Wu (2014), we set the true parameter $(a, b, c)=(3,0.2,0.34), \tau=10, \sigma_{\epsilon}=0.05$, and the initial values $\left(X_{1}(0), X_{2}(0)\right)=(1,-1)$. The sample size is 300 . The empirical sizes and powers with a 0.05 significance level are reported in Table 2.

The performances of $T M_{n}$ and $T^{H}$ are still very well for checking this complex nonlinear ODE model. $G M_{n 1}$ and $G M_{n 2}$ also work well in most cases. Due to the complex interaction between the components of the ODE system, the performances of $G M_{n 1}$ and $G M_{n 2}$ when $(\alpha, \beta)=(0.5,0.5)$ seem totally different compared to the $(\alpha, \beta)=(1,1)$ setting. These simulation results again show the complexity of the ODE testing problem.

Obviously, the size of $I M_{n 1}$ is totally out of control. Some unreported results show that even when the sample size is increased up to 10,000 , the empirical size can then be greatly reduced which suggests consistency, but is still too large to make sense. This reminds that we must be careful to use $I M_{n}$ to check complex nonlinear ODE models. $I M_{n 2}$ performs acceptably 
Table 2: Empirical sizes and powers in Example 2.

\begin{tabular}{ccccccccc}
\hline Hypothesis & $\alpha$ & $\beta$ & $T M_{n}$ & $I M_{n 1}$ & $I M_{n 2}$ & $G M_{n 1}$ & $G M_{n 2}$ & $T^{H}$ \\
\hline$H_{2}$ & 0 & 0 & 0.048 & 0.816 & 0.085 & 0.071 & 0.048 & 0.045 \\
& 0.5 & 0 & 1.000 & 1.000 & 0.070 & 0.131 & 0.048 & 1.000 \\
& 0 & 0.5 & 1.000 & 0.846 & 1.000 & 0.055 & 0.159 & 1.000 \\
& 0.5 & 0.5 & 1.000 & 1.000 & 1.000 & 0.129 & 0.203 & 1.000 \\
& 1 & 0 & 1.000 & 1.000 & 0.089 & 0.514 & 0.059 & 1.000 \\
& 0 & 1 & 1.000 & 0.846 & 1.000 & 0.069 & 0.993 & 1.000 \\
1 & 1 & 1.000 & 1.000 & 0.206 & 0.990 & 0.053 & 1.000 \\
\hline
\end{tabular}

as the hypothetical model is now linear with $(\alpha, \beta)=(0,0)$.

Example 3. The null ODE system is the standard Lotka-Volterra model which is well known for modeling the evolution of prey-predator populations (Goel et al. (1971)). Since the performances of tests are similar to those in the last example, we put the results in Supplementary Material.

Example 4. The hypotheses are the same as in the previous three examples. We only collect the data of the second component: $Y_{i 2}=X_{2}(t)+\epsilon_{i 2}$. The results show $T M_{n}^{o}$ can be applied for partially observed ODE systems. See Supplementary Material for detailed results.

We conclude that $T M_{n}$ and $G M_{n}$ can maintain the significance level with good powers in the conducted simulations. $I M_{n}$ is only usable for checking linear ODEs. $T M_{n}^{o}$ is feasible for partially observed ODEs. Com- 
pared with $T M_{n}, T^{H}$ also performs well in these examples and some other results in Supplementary Material show it may fail to maintain the significance level in the cases with dependent error components or heteroscedasticity where $T M_{n}$ still works. Thus these two tests are complementary to each other, but our test could be more robust against all these scenarios.

\subsection{A real data example}

Apply our tests to a real data set downloaded from Hulin Wu Lab (https://sph.uth.edu/ dotAsset/3ac61148-e59e-493c-bbda-0a38ffe111e5.zip). The data set has been analyzed to show the benefits of differential equation-constrained local polynomial regression for estimating parameters in an ODE model concerning influenza virus-specific effector CD8+ T cells (Ding and Wu (2014)). Here we employ the proposed tests to check the adequacy of this model. See Supplementary Material for details of the model form and the dataset.

The value of $T M_{n}$ is 84.10 and the corresponding $p$-value is about 0 . This suggests that the whole ODE model under the null is not plausible. Next we use $I M_{n}$ and $G M_{n}$ to check each component function. The values of $I M_{n}$ for the three component functions are $(3.17,3.26,4.46)$ and the pvalues are $(0.00077,0.00056,0)$. But as we commented before, this ODE model is not linear and thus, we need to be careful to make a decision only 

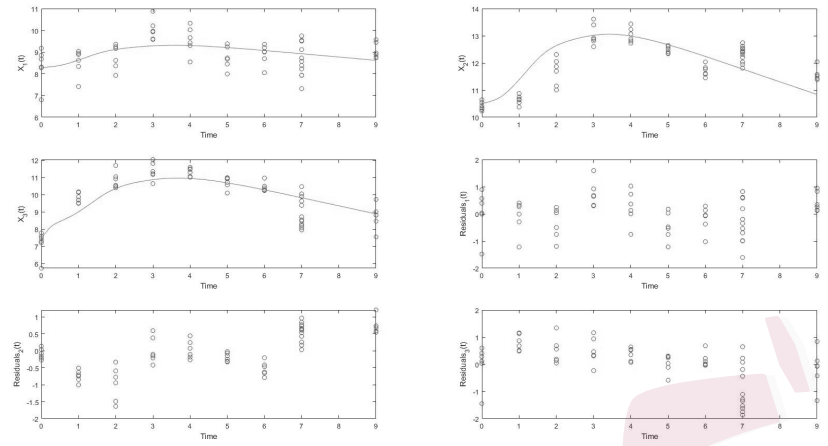

Figure 1: Time course of response and residuals.

based on the result of $I M_{n}$. The values of $G M_{n}$ for the three component functions are $(13.44,2.68,25.96)$ and the $p$-values are $(0,0.0037,0)$. These results again suggest all of the three component functions under the null are not tenable. Therefore, we consider that the models may not well fit the data. On the other hand, we also realize that conclusions which would statistically make sense are only references for the investigations on whether they are of biological meanings. This paper does not discuss this issue.

\section{Discussion}

In this paper, we investigate model checking for parametric ordinary differential equations systems and propose three tests. 
Unlike $T M_{n}, I M_{n}$ and $G M_{n}$ can not deal with partially observed ODE systems. If some components are unmeasured, we can not obtain the kernel estimators to decouple the relationship among different components. Furthermore, the two-step method used to build the tests does not work either for the same reason. The existing estimation methods applied in that case usually need to draw support from the model structure (see, e.g. Dattner (2015)). However, it is difficult to use the information about model structure while eliminating the effects of mixed components and mixed parameters in hypothesis testing. We may need to utilize other methods such as the semiparametric approach used by Hooker (2009) or the profiling method (Ramsay et al. (2007)). How to identify wrongly modelled components in partially observed systems definitely deserves a further study.

We discuss two kinds of alternatives where the disturbances represented as functions of time in the mathematical analysis. However, we have only tried the alternatives that have disturbances on $X^{\prime}(t)$ depending on $X(t)$ in Simulation. In finite sample scenarios, the power may be quite different for the alternatives with other disturbances such as disturbances on $X(t)$ or momentary disturbances not depending on $X(t)$. Thus it is also interesting to try different kinds of alternatives in the further research.

There are other issues worthwhile to investigate in future studies. First, 
we see that $G M_{n}$ performs better than $I M_{n}$, but is still not very satisfactory in some cases. How to solve this problem is a big issue. Second, as seen in the limited simulations, $I M_{n}$ is hard to control the significance level due to its sensitivity to the nonparametric estimator. How to modify it is a nontrivial task. Third, for ODE models, it is also the case where the ODE system is large, that is, $p$ is large. This is a very challenging problem.

Supplementary material. Supplementary material includes the additional conditions, remark of notations, lemmas, technical proofs and some specific details.

Acknowledgement. The study was supported by grants from the University Grants Council of Hong Kong (HKBU12303921), NSFC (12131006) and Science and Technology Innovation Plan of Shanghai (20JC1414200). The authors thank the editor, associate editor and referee for their suggestions that greatly improved an early manuscript.

\section{References}

Brunel, N. J.-B. (2008). Parameter estimation of ode's via nonparametric estimators. Electronic Journal of Statistics 2(0), 1242-1267.

Chen, S. X. and I. van Keilegom (2009). A goodness-of-fit test for parametric and semiparametric models in multiresponse regression. Bernoulli 15(4), 955-976. 
Dattner, I. (2015). A model-based initial guess for estimating parameters in systems of ordinary differential equations. Biometrics $71(4), 1176-1184$.

Dattner, I. and C. A. J. Klaassen (2015). Optimal rate of direct estimators in systems of ordinary differential equations linear in functions of the parameters. Electronic Journal of Statistics 9(2), 1939-1973.

Dette, H. (1999). A consistent test for the functional form of a regression based on a difference of variance estimators. The Annals of Statistics 27(3), 1012-1040.

Ding, A. A. and H. Wu (2014). Estimation of ordinary differential equation parameters using constrained local polynomial regression. Statistica Sinica 24(4), 1613-1631.

FitzHugh, R. (1961). Impulses and physiological states in theoretical models of nerve membrane. Biophysical Journal 1(6), 445-466.

Goel, N. S., S. C. Maitra, and E. W. Montroll (1971). On the volterra and other nonlinear models of interacting populations. Reviews of Modern Physics 43(2), 231-276.

González-Manteiga, W. and R. M. Crujeiras (2013). An updated review of goodness-of-fit tests for regression models. Test 22(3), 361-411.

Hansen, B. E. (2008). Uniform convergence rates for kernel estimation with dependent data. Econometric Theory 24(3), 726-748.

Härdle, W. and E. Mammen (1993). Comparing nonparametric versus parametric regression fits. The Annals of Statistics $21(4), 1926-1947$. 
Hooker, G. (2009). Forcing function diagnostics for nonlinear dynamics. Biometrics 65(3), $928-936$.

Hooker, G. and S. P. Ellner (2015). Goodness of fit in nonlinear dynamics: Misspecified rates or misspecified states? The Annals of Applied Statistics 9(2), 754-776.

Jennrich, R. I. (1969). Asymptotic properties of non-linear least squares estimators. The Annals of Mathematical Statistics 40(2), 633-643.

Khmaladze, E. V. and H. L. Koul (2004). Martingale transforms goodness-of-fit tests in regression models. The Annals of Statistics 32(3), 995-1034.

Lavergne, P. and V. Patilea (2012). One for all and all for one: Regression checks with many regressors. Journal of Business \& Economic Statistics 30(1), 41-52.

Li, L., S. N. Chiu, and L.-X. Zhu (2019). Model checking for regressions: An approach bridging between local smoothing and global smoothing methods. Computational Statistics $\&$ Data Analysis 138, 64-82.

Liang, H. and H. Wu (2008). Parameter estimation for differential equation models using a framework of measurement error in regression models. Journal of the American Statistical Association 103(484), 1570-1583.

Nagumo, J., S. Arimoto, and S. Yoshizawa (1962). An active pulse transmission line simulating nerve axon. Proceedings of the IRE 50(10), 2061-2070.

Ramsay, J. and G. Hooker (2017). Dynamic data analysis. New York, NY: Springer New York. 
Ramsay, J. O., G. Hooker, D. Campbell, and J. Cao (2007). Parameter estimation for differential equations: a generalized smoothing approach. Journal of the Royal Statistical Society: Series B (Statistical Methodology) 69(5), 741-796.

Stute, W. (1997). Nonparametric model checks for regression. The Annals of Statistics 25(2), $613-641$.

Stute, W., S. Thies, and L.-X. Zhu (1998). Model checks for regression: an innovation process approach. The Annals of Statistics 26(5), 1916-1934.

White, H. (1981). Consequences and detection of misspecified nonlinear regression models. Journal of the American Statistical Association 76(374), 419.

Xue, H., H. Miao, and H. Wu (2010). Sieve estimation of constant and time-varying coefficients in nonlinear ordinary differential equation models by considering both numerical error and measurement error. The Annals of Statistics 38(4), 2351-2387.

Zheng, J. X. (1996). A consistent test of functional form via nonparametric estimation techniques. Journal of Econometrics 75(2), 263-289.

Zhu, L.-X. (2003). Model checking of dimension-reduction type for regression. Statistica Sinica 13(2), 283-296.

Hong Kong Baptist University, Hong Kong, China. E-mail: sdqdliuran@life.hkbu.edu.hk Shanghai Normal University, Shanghai 200234, China. E-mail: fangyun0919@shnu.edu.cn Beijing Normal University at Zhuhai, Zhuhai 519085, China. E-mail: lzhu@bnu.edu.cn 